\title{
Impact of Trauma System Structure on Injury Outcomes : A Systematic Review and Meta-Analysis
}

\section{Moore, Lynne}

2018-05

Moore , L , Champion , H , Tardif , P-A , Kuimi , B-L , O'Reilly , G , Leppaniemi , A , Cameron , P , Palmer , C S , Abu-Zidan , F M , Gabbe , B , Gaarder , C , Yanchar , N , Stelfox , H T , Coimbra , R , Kortbeek , J , Noonan , V K, Gunning , A , Gordon , M , Khajanchi , M , Porgo , T V , Turgeon, A F \& Leenen , L 2018 , ' Impact of Trauma System Structure on Injury Outcomes : A Systematic Review and Meta-Analysis ', World Journal of Surgery , vol. 42 , no. 5 , pp. 1327-1339 . https://doi.org/10.1007/s00268-017-4292-0

http://hdl.handle.net/10138/301261

https://doi.org/10.1007/s00268-017-4292-0

unspecified

publishedVersion

Downloaded from Helda, University of Helsinki institutional repository.

This is an electronic reprint of the original article.

This reprint may differ from the original in pagination and typographic detail.

Please cite the original version. 


\title{
Impact of Trauma System Structure on Injury Outcomes: A Systematic Review and Meta-Analysis
}

\author{
Lynne Moore ${ }^{1,2} \cdot$ Howard Champion $^{3} \cdot$ Pier-Alexandre Tardif $^{1,2} \cdot$ Brice-Lionel Kuimi $^{2}$. \\ Gerard O'Reilly $^{4,8} \cdot$ Ari Leppaniemi ${ }^{5}$ - Peter Cameron ${ }^{4,8} \cdot$ Cameron S. Palmer $^{6}$. \\ Fikri M. Abu-Zidan ${ }^{7}$ - Belinda Gabbe ${ }^{8}$ - Christine Gaarder ${ }^{9}$ Natalie Yanchar $^{10}$. \\ Henry Thomas Stelfox ${ }^{11} \cdot$ Raul Coimbra ${ }^{12} \cdot$ John Kortbeek $^{13} \cdot$ Vanessa K. Noonan $^{14}$. \\ Amy Gunning ${ }^{15}$ - Malcolm Gordon ${ }^{16} \cdot$ Monty Khajanchi $^{17} \cdot$ Teegwendé V. Porgo ${ }^{1,2}$. \\ Alexis F. Turgeon ${ }^{1,2} \cdot$ Luke Leenen $^{15}$ - On behalf of the International Injury Care Improvement Initiative
}

Published online: 25 October 2017

(C) Société Internationale de Chirurgie 2017

\begin{abstract}
Background The effectiveness of trauma systems in decreasing injury mortality and morbidity has been well demonstrated. However, little is known about which components contribute to their effectiveness. We aimed to systematically review the evidence of the impact of trauma system components on clinically important injury outcomes.

Methods We searched MEDLINE, EMBASE, Cochrane CENTRAL, and BIOSIS/Web of Knowledge, gray literature and trauma association Web sites to identify studies evaluating the association between at least one trauma system component and injury outcome. We calculated pooled effect estimates using inverse-variance random-effects models. We evaluated quality of evidence using GRADE criteria.

Results We screened 15,974 records, retaining 41 studies for qualitative synthesis and 19 for meta-analysis. Two recommended trauma system components were associated with reduced odds of mortality: inclusive design (odds ratio $[\mathrm{OR}]=0.72[0.65-0.80])$ and helicopter transport $(\mathrm{OR}=0.70[0.55-0.88])$. Pre-Hospital Advanced Trauma Life Support was associated with a significant reduction in hospital days (mean difference [MD] $=5.7$ [4.4-7.0]) but a nonsignificant reduction in mortality $(\mathrm{OR}=0.78$ [0.44-1.39]). Population density of surgeons was associated with a nonsignificant decrease in mortality ( $\mathrm{MD}=0.58[-0.22$ to 1.39$]$ ). Trauma system maturity was associated with a significant reduction in mortality $(\mathrm{OR}=0.76[0.68-0.85])$. Quality of evidence was low or very low for mortality and healthcare utilization.

Conclusions This review offers low-quality evidence for the effectiveness of an inclusive design and trauma system maturity and very-low-quality evidence for helicopter transport in reducing injury mortality. Further research should evaluate other recommended components of trauma systems and non-fatal outcomes and explore the impact of system component interactions.
\end{abstract}

The International Injury Care Improvement Initiative (IICII) is a global effort of over 60 injury care experts, harnessing national capabilities in injury control from 30 countries in pursuit of our mission to reduce the global burden of injuries.

Electronic supplementary material The online version of this article (doi:10.1007/s00268-017-4292-0) contains supplementary material, which is available to authorized users.

Lynne Moore

lynne.moore@fmed.ulaval.ca

1 Department of Anesthesiology and Critical Care Medicine, Division of Critical Care Medicine, Université Laval,

Québec, QC, Canada

\section{Introduction}

Injury is the leading cause of death under 40 years of age, the leading cause of loss of active life years, and is second only to cardiovascular diseases in terms of healthcare costs

2 Axe Santé des Populations et Pratiques Optimales en Santé (Population Health and Optimal Health Practices Research Unit), Traumatologie - Urgence - Soins intensifs (Trauma Emergency - Critical Care Medicine), CHU de Québec Université Laval Research Center (Enfant-Jésus Hospital), Québec, QC, Canada 
in high-income countries [1]. Low- to middle-income countries carry more than $90 \%$ of the fatal injury burden [2]. Important reductions in injury mortality, disability, and costs have been achieved in many healthcare jurisdictions with the introduction of trauma systems [3, 4].

There are multiple specific definitions of a trauma system, but broadly, it is an organized, regional, multidisciplinary response to injury $[5,6]$. Many injury organizations, including the World Health Organization (WHO) [7] and the American College of Surgeons (ACS) [8], provide consensus-based recommendations on the structure of trauma systems. Consequently, system components such as pre-hospital triage and transport protocols, accreditation and designation and benchmarking activities as well as their level of integration vary significantly across trauma systems [9]. The effectiveness of trauma systems has now been well established; they have been estimated to lead to a $15 \%$ reduction in the odds of mortality [10] and have been associated with reductions in disability and costs [11]. However, there is still a major knowledge gap on which components of a trauma system contribute to their effectiveness. Given the multitude of recommended trauma system components and the fact that they are largely based on expert consensus, there is an urgent need to build an evidence base to guide budget-constrained policy-makers, particularly in low- and middle-income countries.

Our aim was to systematically review evidence of the impact of trauma system components on clinically important injury outcomes including mortality, function, disability, quality of life, and resource utilization.

3 Department of Surgery, University of the Health Sciences, Annapolis, MD, USA

4 Emergency and Trauma Centre, The Alfred Hospital, Melbourne, Australia

5 Abdominal Center, Helsinki University hospital, Helsinki, Finland

6 Trauma Service, Royal Children's Hospital, Melbourne, Australia

7 Department of Surgery, College of Medicine and Health Sciences, United Arab Emirates University, Al-Ain, United Arab Emirates

8 School of Public Health and Preventive Medicine, Monash University, Melbourne, Australia

9 Department of Traumatology, Oslo University Hospital Ulleval, Oslo, Norway

10 Department of Surgery, Dalhousie University, Halifax, NS, Canada

\section{Materials and methods}

We conducted a systematic review in accordance with Cochrane guidelines [12]. The review is presented using the structure suggested in Preferred reporting items for systematic review and meta-analysis (PRISMA) 2015 [13]. Our systematic review protocol was registered with the International Prospective Register of Systematic Reviews (PROSPERO) on June 20, 2016 (\#42016041336) and published in Systematic Reviews [14].

\section{Eligibility criteria}

\section{Study designs}

We considered randomized and non-randomized controlled trials (RCTs) including cluster RCT, interrupted time series studies, controlled before-after studies, and prospective or retrospective observational studies. We also included studies based on qualitative methods (e.g., preventable death determined by expert consensus).

\section{Participants}

We included studies based on injury populations at large as well as studies evaluating population-based injury outcomes. No restrictions were placed on age, injury type, or injury severity. Studies based exclusively on combat injuries, isolated fractures following low falls, burns, bites, foreign bodies or late effects of injuries were excluded.

\section{Interventions}

We included studies evaluating the effectiveness of trauma system components, i.e., organizational-level structural

11 Departments of Critical Care Medicine, Medicine and Community Health Sciences, O'Brien Institute for Public Health, University of Calgary, Calgary, Canada

12 Division of Trauma, Surgical Critical Care, Burns, and Acute Care Surgery, University of California, San Diego Health System, San Diego, CA, USA

13 Department of Surgery, Division of General Surgery and Division of Critical Care, University of Calgary, Calgary, $\mathrm{AB}$, Canada

14 Rick Hansen Institute, Vancouver, BC, Canada

15 Department of Surgery, University Medical Center Utrecht, Utrecht, The Netherlands

16 Department of Emergency Medicine, University of Glasgow, Glasgow, UK

17 Seth G.S. Medical College and KEM Hospital, Mumbai, India 
interventions (single or multiple) targeting tertiary injury prevention (optimal treatment following injury). Interventions were classified using an adaptation of WHO and ACS categories, i.e., oversight, pre-hospital care, definitive care, rehabilitation, and evaluation $[7,8]$.

\section{Comparators}

Studies comparing a single or multiple organizational-level structural intervention to either (1) usual trauma system structure or (2) an alternative organizational structure were eligible. In order to be as inclusive as possible, and given the variation in definitions of trauma systems, we included studies based on authors' definition of a trauma system. No restrictions were based on the country or the regulatory nature of the trauma system (e.g., mandatory, nonmandatory, or volunteer). Studies comparing healthcare jurisdictions with a trauma system to those without organized trauma care were not included as evidence of the global effectiveness of trauma systems has already been reviewed [10].

\section{Outcome measures}

Primary endpoints of interest were clinically important outcomes, established by the study steering committee, including mortality, function, disability, and quality of life. Secondary outcomes were adverse events, healthcare utilization, and costs. No restrictions were imposed on the follow-up of patients for the evaluation of injury outcomes.

\section{Information sources}

The search strategy was designed to minimize publication bias, including geographical bias. We systematically searched MEDLINE, EMBASE, Cochrane CENTRAL, and BIOSIS/Web of Knowledge databases from their inception up to March 2, 2017. Unpublished clinical studies were searched using ClinicalTrials and the ISRCTN registry. We consulted thesis repositories to identify additional studies, including Thesis portal Canada, EtHOS, DART-Europe E-Theses Portal, and ProQuest Dissertations \& Theses Global. We also searched the Web sites of key healthcare organizations (WHO, public health agencies) and injury organizations including the American College of Surgeons, the Trauma Association of Canada, the International Association for Trauma Surgery and Intensive Care, the Australasian Trauma Society and the Trauma Audit Research Network. We then screened references of included articles and abstracts of major injury conferences including the International Surgical Week, World Congress of Surgery, American Association for the Surgery of Trauma congress, European Congress of Trauma and
Emergency Surgery, Western Trauma Association congress, World Trauma Congress, Eastern Society for the Surgery of Trauma Congress, Trauma Association of Canada annual meeting, and Australasian Trauma Society Congress.

\section{Search strategy}

We developed a rigorous systematic search strategy with a health sciences information specialist who has systematic review experience (MS) using published guidelines of The Cochrane Collaboration (see Online resource 1 for the MEDLINE search strategy via PubMed) [15]. The strategy was developed using keywords and MeSH (MEDLINE) or EMTREE (EMBASE). To be as inclusive as possible, we limited the search strategy to terms covering the concept of «trauma system》. Keywords were elaborated by co-investigators and collaborators with methodological and clinical expertise. This search strategy was then adapted to the other databases.

\section{Study records}

\section{Data management}

Citations were managed using EndNote software (version X7.0.1, New York City: Thomson Reuters, 2011). Duplicates were identified and eliminated using electronic and manual screening. No multiple publications based on the same data were identified.

\section{Selection process}

Pairs of reviewers (LM, PAT, TVP) independently evaluated citations for potential inclusion by screening titles and abstracts and assessed full publications to determine eligibility for final inclusion. To ensure high agreement on study eligibility, three samples of 500 citations were independently and consecutively assessed by each reviewer. Between each assessment, results were discussed to reach a consensus on the interpretation of inclusion criteria. Any further disagreement on study eligibility was resolved by consensus and a third reviewer (HC) adjudicated when necessary.

\section{Data collection}

A standard electronic data abstraction form was developed and piloted on a representative sample of five studies. Two reviewers (LM and PAT) with methodological and content expertise independently extracted information on study setting and design, study population, interventions, outcomes, measures of association with standard errors, and 
risk adjustment. If information relating to the above-mentioned elements was missing, study authors were contacted by email (up to three attempts) for further clarifications. Abstracts from conference proceedings were included if they provided information on all of the above.

\section{Risk of bias in individual studies}

Risk of bias was evaluated using a study-specific adaptation of the ROBINS-I tool [16]. This tool evaluates baseline and time-varying confounding, co-interventions, selection bias, classification bias (intervention), missing data, and bias in outcome measurement. Two reviewers (LM and PAT) independently evaluated the risk of bias and rated studies. Disagreement was resolved using arbitration by a third reviewer (HC).

\section{Assessment of heterogeneity}

Statistical heterogeneity was measured using $\mathrm{I}^{2}$ statistics and was interpreted as low from 0 to $40 \%$, moderate from 30 to $60 \%$, substantial from 50 to $90 \%$, and considerable from 75 to $100 \%$ as recommended in the Cochrane handbook [12].

\section{Data synthesis}

If two or more studies had evaluated the same intervention and the same outcome, we calculated pooled effect estimates and their 95\% confidence intervals (CI) using inverse-variance random-effects models (DerSimonian and Laird) [17] adapted to the scale of measurement. We used Review Manager (RevMan), version 5.3 Copenhagen: The Nordic Cochrane Centre, The Cochrane Collaboration, 2014.

\section{Subgroup and sensitivity analyses}

Due to lack of data and the small number of studies that assessed the same outcome for a given component, we could not perform planned subgroup analyses (age group, injury severity, injury type, length of follow-up, and World Bank country economic classifications). However, when the number of studies available for analyses was sufficient $(\geq 2)$, we conducted sensitivity analyses excluding studies of low methodological quality.

\section{Publication bias}

We generated funnel plots adapted to the scale of measurement to evaluate the risk of publication bias.

\section{Quality of evidence}

Two subject-content experts (LM and GOR) independently evaluated quality of evidence for each intervention-outcome evaluation included in meta-analysis using the Grading of Recommendations Assessment, Development and Evaluation (GRADE) working group methodology [18].

\section{Results}

\section{Study identification and selection}

The search strategy retrieved 24,758 citations, and a total of 15,974 titles were screened after removing duplicates (Fig. 1). One hundred and thirty-four full texts were assessed, of which 33 were deemed eligible. One conference abstract was included [19], and an additional seven articles were found through gray literature and hand searching of the references of included studies. In total, 41 studies were selected for the systematic review, of which 19 were included in the meta-analysis.

\section{Study characteristics}

All studies included were written in English, but study cohorts originated from thirteen different countries, six of which were situated in Europe, three in North America, three in Asia, and one in Oceania (Online resource 2). Three studies $[20,21]$ were conducted in low- or middleincome countries [22], and five (12\%) studies compared cohorts from two different countries [20, 23-26]. Most studies $(88 \%)$ used a prospective or retrospective observational design, but there were three controlled beforeafter studies [27-29], one interrupted time series [30], and one cluster randomized controlled trial [31]. Data collection spanned from 1990 [32] to 2013 [33] and included between 35 [34] and 857,534 [35] patients from 1 [32] to $>600$ [26] trauma centers. Mean age of patients ranged from 26 [36] to 57 [37] years (excluding three studies restricted to pediatric trauma) $[28,38,39]$. Mean ISS (when provided) varied between 10 [34] and 29 [24]. Five studies used states $[33,40]$ or counties $[19,41,42]$ as their unit of analysis.

\section{Risk of bias}

Eighty percent $(n=32)$ of the studies had a moderate or serious risk of bias in relation to confounding $(32,80 \%)$ and classification of interventions $(35,88 \%$ ) (Online resource 3 ). In the domains associated with the selection of study participants and deviations from intended 


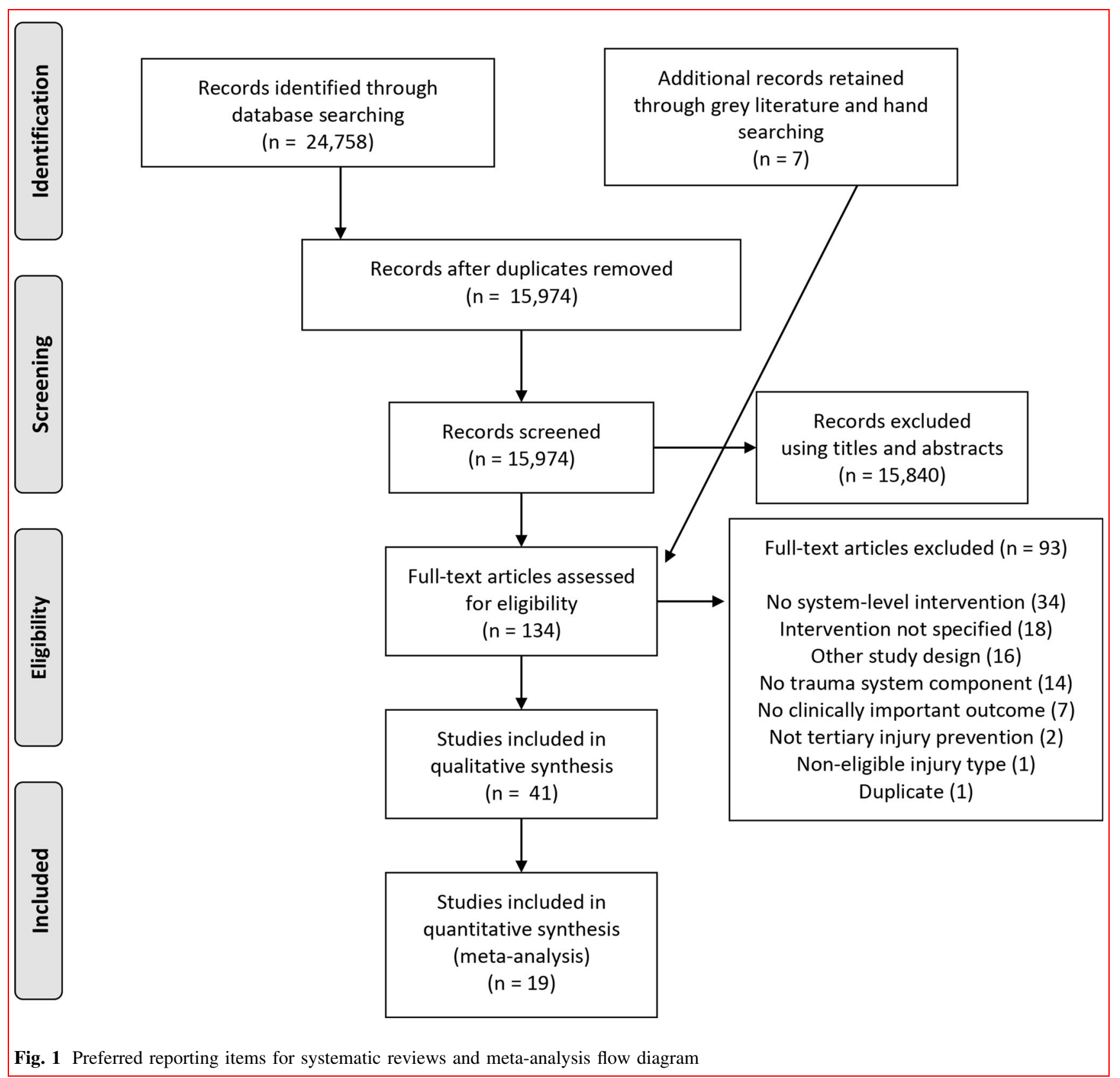

interventions, $26(65 \%)$ and $24(60 \%)$ studies had low or moderate risk of bias, respectively. Information on missing data was often not reported (40\%), and among those studies with missing data, it was rarely handled appropriately (i.e., imputation methods which account for the uncertainty of missing data, 15\%). Finally, 40 (100\%) studies had a moderate or low risk of bias for the measurement of outcome and $38(95 \%)$ for selective reporting of results.

Visual inspection of funnel plots suggested no significant bias for odds ratios (OR) (16 studies; Online resource 4 , left side) but a possible publication bias in favor of studies that reported a positive intervention effect for mean differences (four studies; Online resource 4, right panel).
Studies with higher standard errors were slightly more likely to report a positive intervention effect on either scale, suggesting a small-study effect.

\section{Impact of trauma system components}

Overall, 84 assessments of the association between trauma system components and outcomes were reported (Table 1). Mortality alone represented $66 \%$ of all assessments, whereas healthcare utilization and function and disability represented 21 and $7 \%$, respectively. The impact of trauma system components on quality of life, costs, and adverse events was each assessed twice or less. The most common 
Table 1 Number of evaluations of the effectiveness of recommended trauma system components ${ }^{\mathrm{a}}$ according to clinically important outcomes

\begin{tabular}{|c|c|c|c|c|c|c|}
\hline \multirow[t]{2}{*}{ Trauma system component subcomponent ${ }^{a}$} & \multicolumn{6}{|l|}{ Outcomes (number of studies) } \\
\hline & Mortality & $\begin{array}{l}\text { Function } \\
\text { and } \\
\text { disability }\end{array}$ & $\begin{array}{l}\text { Quality } \\
\text { of life }\end{array}$ & $\begin{array}{l}\text { Adverse } \\
\text { events }\end{array}$ & $\begin{array}{l}\text { Healthcare } \\
\text { utilization }\end{array}$ & Costs \\
\hline \multicolumn{7}{|l|}{ Oversight } \\
\hline \multicolumn{7}{|l|}{ Disaster planning } \\
\hline Lead agency & $1[43]$ & & & & & \\
\hline \multicolumn{7}{|l|}{ Trauma services medical director } \\
\hline \multicolumn{7}{|l|}{ Trauma system advisory committee } \\
\hline Trauma system plan & $1[43]$ & & & & & \\
\hline \multicolumn{7}{|l|}{ Pre-hospital care } \\
\hline \multicolumn{7}{|l|}{ Communication between EMS and hospitals } \\
\hline \multicolumn{7}{|l|}{ Emergency services medical director } \\
\hline EMS treatment protocols & $6[21,27,32,61]$ & $3[27]$ & & & $3[27,32]$ & \\
\hline EMS transport system & $3[29,62]$ & & & & & \\
\hline Pre-hospital major trauma definition & $1[43]$ & & & & & \\
\hline Triage and transport protocols & $3[26,31,43]$ & $1[31]$ & $1[31]$ & & $2[28]$ & \\
\hline \multicolumn{7}{|l|}{ Definitive care } \\
\hline Communication between transferring hospitals & $1[43]$ & & & & & \\
\hline Facility designation through an accreditation agency & $1[43]$ & & & & & \\
\hline Inclusive design & $6[35,43,63-66]$ & & & & $1[66]$ & $1[64]$ \\
\hline Interfacility transfer agreements/protocols & $2[43]$ & & & & & \\
\hline Relative location of trauma centers & $7[19,30,33,37,40,67,68]$ & & & & & \\
\hline Other & $2[25,34]$ & & & & $2[25]$ & \\
\hline \multicolumn{7}{|l|}{ Rehabilitation } \\
\hline \multicolumn{7}{|l|}{ Human resources } \\
\hline \multicolumn{7}{|l|}{ Educational preparation } \\
\hline Workforce resources & $4[33,41,42]$ & & & & & \\
\hline \multicolumn{7}{|l|}{ Evaluation } \\
\hline Benchmarking & $1[69]$ & & & $1[69]$ & $3[69]$ & \\
\hline \multicolumn{7}{|l|}{ Data collection-trauma registries } \\
\hline \multicolumn{7}{|l|}{ Injury surveillance } \\
\hline \multicolumn{7}{|l|}{$\begin{array}{l}\text { Integration of evaluation throughout the care } \\
\text { continuum }\end{array}$} \\
\hline \multicolumn{7}{|l|}{ Interdisciplinary review committee } \\
\hline \multicolumn{7}{|l|}{ Research } \\
\hline \multicolumn{7}{|l|}{ Other } \\
\hline System maturity & $5[4,11,39,70,71]$ & $1[11]$ & & $1[4]$ & $4[4,71]$ & \\
\hline Multiple non-specified & $2[23,24]$ & & & & $2[72]$ & \\
\hline Multiple specified & $9[20,36,38,43,73-75]$ & $1[38]$ & & $1[20]$ & $1[38]$ & \\
\hline
\end{tabular}

${ }^{\mathrm{a}}$ Trauma system components recommended by the American College of Surgeons [8] and the World Health Organization [7]

system components evaluated were in pre-hospital care and definitive care (23 evaluations each). We identified 11 evaluations of trauma system maturity and 16 evaluations of specified or non-specified multiple interventions. Almost half (11 out of 24) of the recommended trauma system components were not evaluated for any outcome. No interventions related to rehabilitation were reported for any outcome.

\section{Interventions}

Pre-Hospital Advanced Trauma Life Support (PH-ATLS) was associated with a decrease in mortality in two studies but an increase in another, leading to a nonsignificant OR with considerable heterogeneity (Fig. 2). In contrast, PHATLS was associated with a significant decrease in hospital LOS (Fig. 3). Similarly, the overall effect of 
helicopter transport suggested a significant decrease in mortality (Fig. 2). The impact of triage and transport protocols on mortality was inconclusive [31, 43]. All studies evaluating the impact of an inclusive trauma system design reported a reduction in mortality, leading to a significant OR characterized by high precision and low heterogeneity. Likewise, all studies but one [11] that assessed the association between trauma system maturity and mortality reported a statistically significant OR with a narrow IC and moderate heterogeneity. Finally, increased population density of surgeons was associated with a statistically significant decrease in mortality in both studies included in the analysis. In a fixed-effects model, the overall mean difference was significant (MD -0.22 [95\% CI: -0.28 to $-0.16]$ ), but the association did not remain statistically significant in a random-effects model due to considerable heterogeneity (Fig. 3).

Among the 26 quantitative assessments reported in studies not included in meta-analyses, 13 (65\%) were statistically significant and all but one [24] suggested that the trauma system intervention was associated with a reduction in mortality (Online resource 5). In addition to lead agency, designation of trauma centers, and inclusiveness of trauma systems, all interventions related to the relative location of trauma centers were associated with a statistically significant reduction in mortality.

\section{Quality of evidence}

Among associations that were statistically significant in meta-analyses, GRADE quality of evidence was low for an inclusive design and system maturity (mortality), very low for helicopter transport (mortality), and very low for $\mathrm{PH}-$ ATLS (healthcare utilization; Table 2). Elsewhere, quality of evidence was low for the density of surgeons (mortality) and very low for PH-ATLS and pre-hospital triage criteria (mortality).

\section{Sensitivity analyses}

The number of studies was sufficient to conduct sensitivity analysis on risk of bias for inclusive design and maturity of trauma systems. Restricting analyses to studies of high methodological quality did not lead to any significant change in pooled estimates (Online resource 6).

\section{Discussion}

The results of this systematic review suggest that overall, the configuration of trauma systems influences clinically important injury outcomes. Meta-analyses offer low-quality evidence of the effectiveness of an inclusive design and trauma system maturity and very-low-quality evidence for helicopter transport. Effect sizes were similar across trauma system components, suggesting a reduction of around $30 \%$ in the odds of mortality. There is weaker evidence that population density of surgeons is associated with a reduction in mortality. Results also suggest that the relative location of trauma centers is an important factor, but included studies were too heterogeneous to perform a meta-analysis.

Our results offer evidence that trauma systems organized to get the right patient to the appropriate acute care facility in a timely manner offer effective injury control. First, all studies in our review on trauma system inclusiveness (five studies) and the relative location of trauma centers (six studies) reported significant associations with mortality. However, these results should be interpreted in light of studies demonstrating the negative correlation between patient volume and mortality [44], suggesting that the balance between inclusiveness and sufficient volume is not yet well understood. Second, we did not find evidence that PH-ATLS, implying longer on-scene times, is associated with reduced mortality. This is in line with another systematic review [45] on the effectiveness of PH-ATLS training and the controlled before-after trial of Stiell et al. [27], which showed no survival benefit following the introduction of an PH-ATLS program and even observed higher mortality post-implantation in patients with severe traumatic brain injury. Furthermore, a comparison of the pre-hospital trauma systems in Germany and the Netherlands showed lower mortality in a system based on fast and continuous pre-hospital treatment to reduce on-scene time [26]. Third, our review provides evidence that helicopter transport within trauma systems reduces injury mortality. However, this evidence should be interpreted with caution as it was of very low quality, in line with previous reviews that have offered inconsistent evidence of the effectiveness and cost-effectiveness of rotary-wing transport [46-50]. We did not identify sufficient evidence to conclude on the effectiveness of pre-hospital triage. Studies on pre-hospital triage mostly evaluate the sensitivity and specificity of triage tools for identifying patients with major trauma, defined using measures of injury severity or need for highlevel care (e.g., surgery, ICU admission) [51]. Our metaanalysis was based on only two low-powered studies, in line with another review that identified no studies meeting their inclusion criteria [52]. We concur with the authors of this review, who conclude that there is an important evidence gap on the effectiveness of pre-hospital triage for improving clinically important injury outcomes [52].

We identified two population-based studies offering evidence that high population density of (neuro)surgeons is associated with lower mortality. Our pooled estimate was statistically significant in a fixed-effects model but not in 


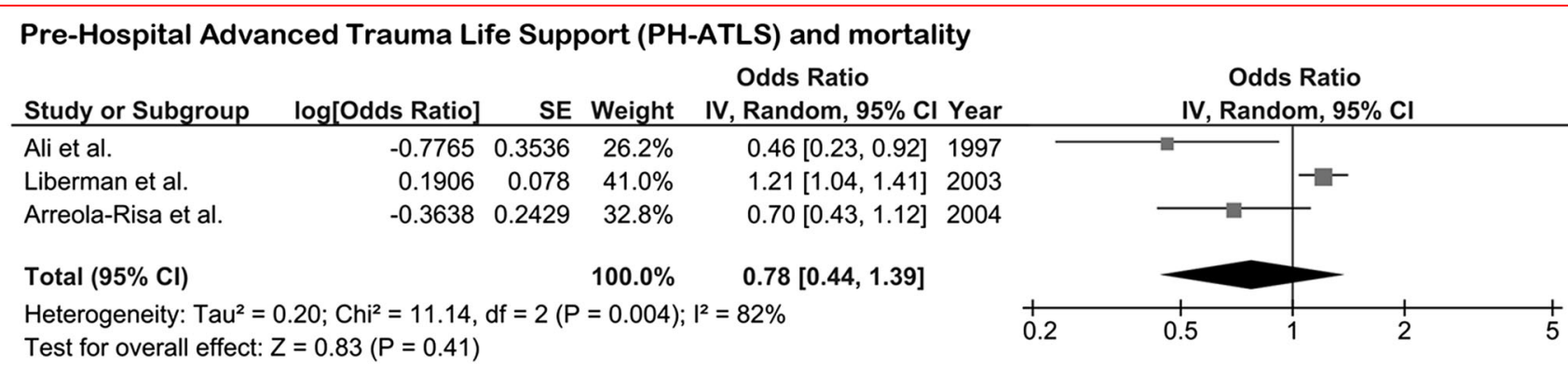

Helicopter transport and mortality

\begin{tabular}{|c|c|c|c|c|c|}
\hline Study or Subgroup & log[Odds Ratio] & SE & Weight & $\begin{array}{c}\text { Odds Ratio } \\
\text { IV, Random, } 95 \% \mathrm{Cl}\end{array}$ & Year \\
\hline Schiller et al. & -0.3147 & 0.1289 & $83.9 \%$ & $0.73[0.57,0.94]$ & 2009 \\
\hline Hessefeldt et al. & -0.5978 & 0.2947 & $16.1 \%$ & $0.55[0.31,0.98]$ & 2013 \\
\hline Total (95\% Cl) & & & $100.0 \%$ & $0.70[0.55,0.88]$ & \\
\hline
\end{tabular}
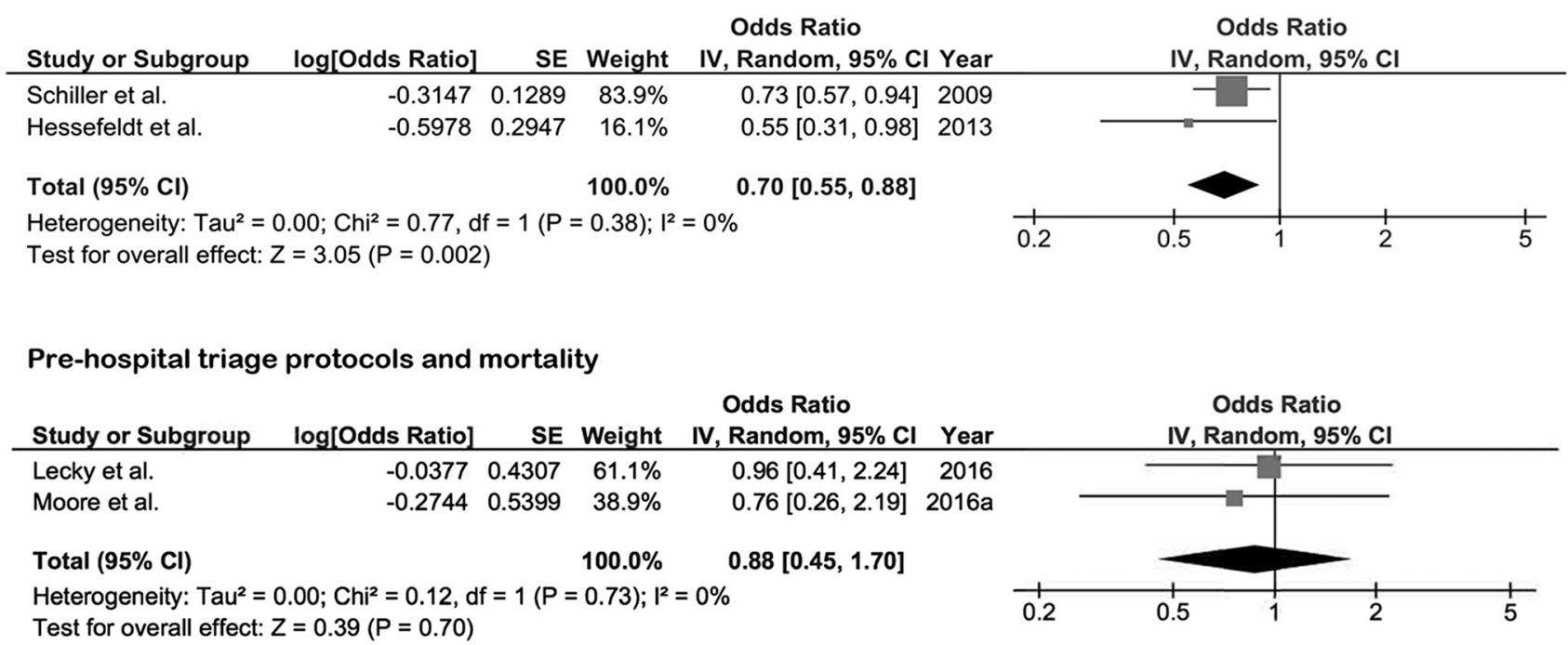

Inclusive design of trauma systems and mortality

\begin{tabular}{|c|c|c|c|c|c|c|c|c|c|}
\hline Study or Subgroup & log[Odds Ratio] & SE & Weight & $\begin{array}{l}\text { Odds Ratio } \\
\text { IV, Random, } 95 \% \mathrm{Cl}\end{array}$ & Year & & $\begin{array}{r}\text { Odds } \\
\text { IV, Rando }\end{array}$ & $\begin{array}{l}\text { Ratio } \\
\text { om, } 95 \% \mathrm{Cl}\end{array}$ & \\
\hline Utter et al. & -0.2614 & 0.1282 & $15.0 \%$ & $0.77[0.60,0.99]$ & 2006 & & & & \\
\hline Arthur et al. & -0.3285 & 0.1521 & $10.6 \%$ & $0.72[0.53,0.97]$ & 2009 & & & & \\
\hline Hamlat et al. & -0.3638 & 0.1087 & $20.8 \%$ & $0.70[0.56,0.86]$ & 2012 & & $\longrightarrow$ & & \\
\hline Vanni et al. & -0.3174 & 0.0742 & $44.6 \%$ & $0.73[0.63,0.84]$ & 2012 & & - & & \\
\hline Moore et al. & -0.3857 & 0.1652 & $9.0 \%$ & $0.68[0.49,0.94]$ & $2016 a$ & & & & \\
\hline Total $(95 \% \mathrm{Cl})$ & & & $100.0 \%$ & $0.72[0.65,0.80]$ & & & & & \\
\hline \multicolumn{6}{|c|}{$\begin{array}{l}\text { Heterogeneity: } \mathrm{Tau}^{2}=0.00 ; \mathrm{Chi}^{2}=0.52, \mathrm{df}=4(\mathrm{P}=0.97) ;\left.\right|^{2}=0 \% \\
\text { Test for overall effect: } Z=6.58(P<0.00001)\end{array}$} & $\frac{+}{0.2}$ & 0.5 & 2 & 5 \\
\hline
\end{tabular}

\section{Trauma system maturity and mortality}

\begin{tabular}{|c|c|c|c|c|c|c|c|c|c|}
\hline Study or Subgroup & log[Odds Ratio] & SE & Weight & $\begin{array}{l}\text { Odds Ratio } \\
\text { IV, Random, } 95 \% \mathrm{Cl}\end{array}$ & Year & & $\begin{array}{r}\text { Odds } \\
\text { IV, Randc }\end{array}$ & $\begin{array}{l}\text { Ratio } \\
\text { om, } 95 \% \mathrm{Cl}\end{array}$ & \\
\hline Cameron et al. & -0.478 & 0.13 & $13.7 \%$ & $0.62[0.48,0.80]$ & 2008 & & $\longrightarrow$ & & \\
\hline Deasy et al. & -0.1393 & 0.0659 & $27.8 \%$ & $0.87[0.76,0.99]$ & 2012 & & & & \\
\hline Gabbe et al. & -0.207 & 0.1137 & $16.3 \%$ & $0.81[0.65,1.02]$ & 2012 & & & & \\
\hline Moore et al. & -0.3285 & 0.0725 & $25.9 \%$ & $0.72[0.62,0.83]$ & 2015 & & $\rightarrow$ & & \\
\hline Moore et al. & -0.33 & 0.1139 & $16.3 \%$ & $0.72[0.58,0.90]$ & 2017 & & 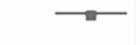 & & \\
\hline Total $(95 \% \mathrm{Cl})$ & & & $100.0 \%$ & $0.76[0.68,0.85]$ & & & & & \\
\hline \multicolumn{6}{|c|}{$\begin{array}{l}\text { Heterogeneity: } \mathrm{Tau}^{2}=0.01 ; \mathrm{Chi}^{2}=7.65, \mathrm{df}=4(\mathrm{P}=0.11) ; \mathrm{I}^{2}=48 \% \\
\text { Test for overall effect: } Z=4.76(P<0.00001)\end{array}$} & 0.2 & 0.5 & 2 & 5 \\
\hline
\end{tabular}

Fig. 2 Meta-analyses of the association between trauma system components and clinically important outcomes (multiplicative scale) 


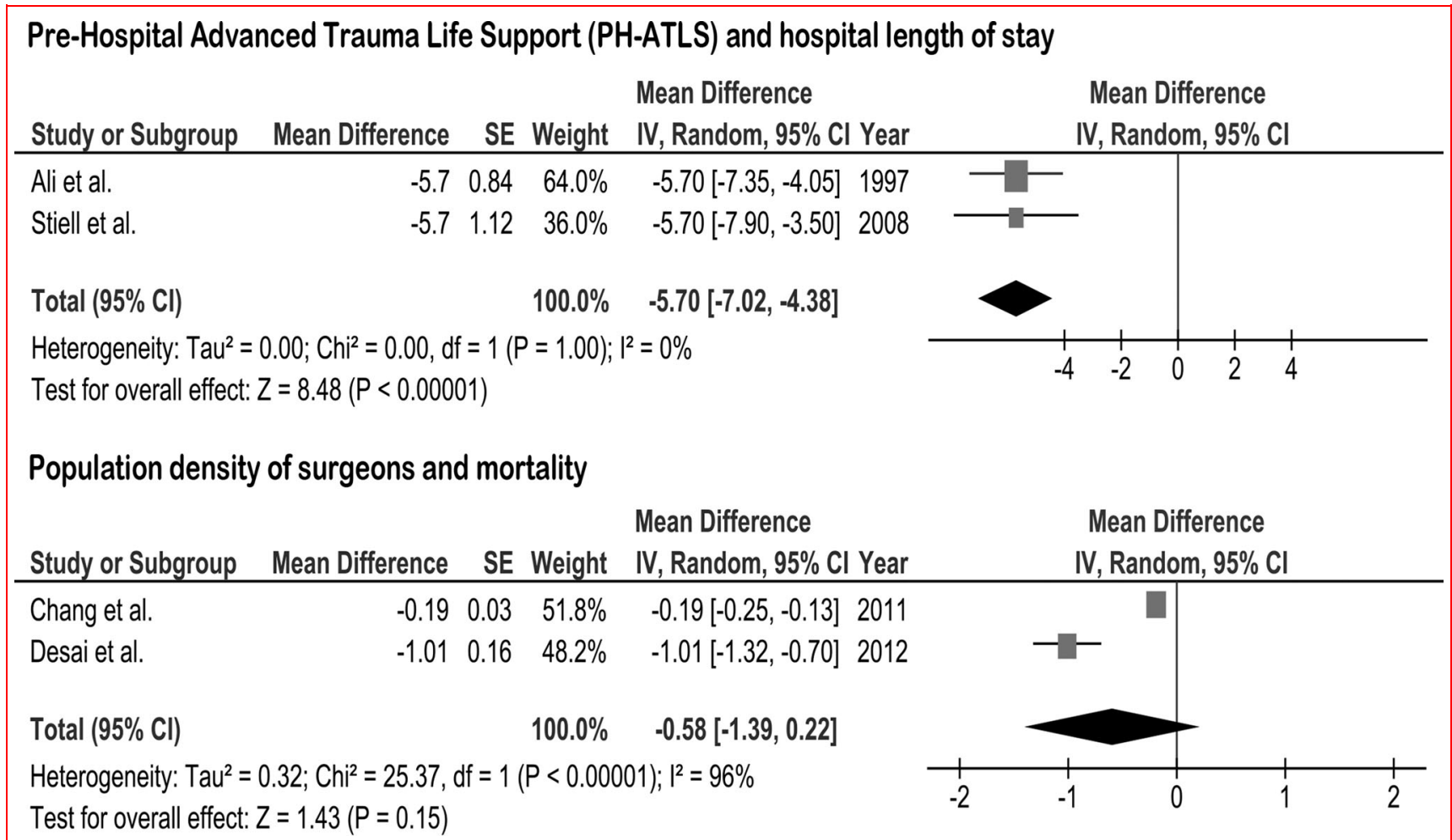

Fig. 3 Meta-analyses of the association between trauma system components and clinically important outcomes (additive scale)

the random-effects model due to heterogeneity. Previous research has suggested that surgical workforce is an important factor globally and for specific diagnoses, particularly in low- and middle-income countries [53-57]. In addition, US counties with 24-hour coverage of general, orthopedic, and neurosurgeons have been reported to have lower motor vehicle collision mortality than those without continuous coverage [58].

Globally, trauma systems or designated trauma centers have been estimated to reduce the odds of mortality by $15 \%$ when compared to non-designated hospitals or health systems with no formal trauma system in North America [10]. Similarly, regionalized trauma systems have been associated with a $16 \%$ reduction in mortality odds compared to healthcare jurisdictions with no trauma system [59]. However, our results on trauma system maturity support the hypothesis that trauma systems are not fully effective until up to 10 years after their implementation $[4,60]$ and suggest that these estimates of mortality reduction are probably underestimated. Finally, interventions related to benchmarking were not associated with any outcome but all evaluations were conducted in a single study.

\section{Strengths and limitations}

This systematic review represents an important step toward identifying the components of trauma systems that drive optimal patient outcomes. Our review was based on the highest methodological standards and was informed by experts involved at all levels and in all phases of the continuum of trauma care from 30 high-, middle-, and lowincome countries. Our review does have limitations which restrict our ability to draw firm conclusions. First, the methodological quality of included studies was generally poor, and the quality of evidence for interventions included in meta-analysis was low or very low. This is partly due to the fact that most studies were observational and failed to adjust for important confounders. However, as our review was based on interventions implemented on a system level, we avoided the common problem of bias by indication encountered in studies evaluating interventions applied at a patient level. Second, we did not obtain data on all clinically important outcomes. The data available on adverse events, function, disability, and quality of life were scarce and could not be used for meta-analysis. Quality of evidence evaluations was therefore restricted to mortality. Third, we could only conduct meta-analysis for six of 24 recommended trauma system components. For 11 components, no studies were identified and for seven, available 


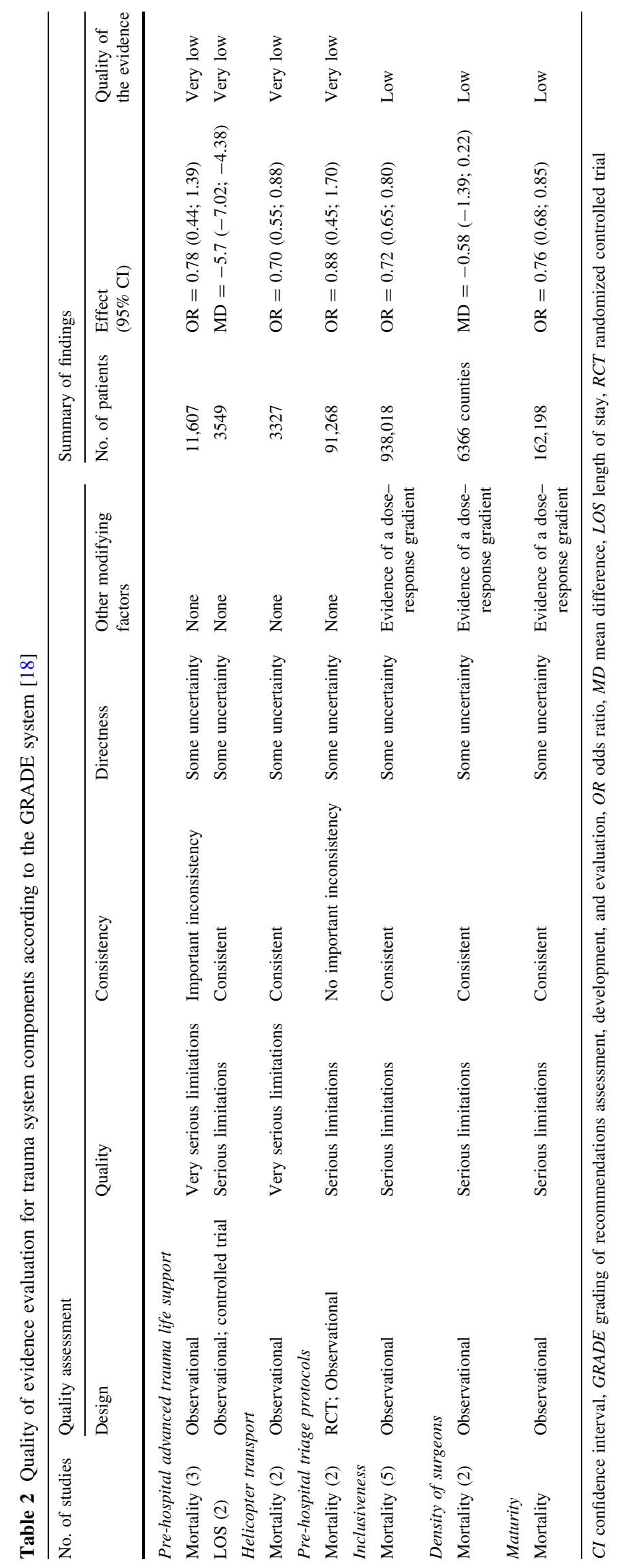


data did not allow meta-analysis. Fourth, we cannot make recommendations according to GRADE criteria as they require data to evaluate trade-offs between benefits and harms, net benefits and costs [18]. Fifth, very little evidence was generated for low- or middle countries. Information on trauma system component effectiveness is even more crucial in such resource-constrained environments, and many of these countries are in the process of implementing trauma systems [7]. Finally, trauma systems are complex interventions, involving interplay between components of care. As such, 13 out of 20 studies evaluated multiple specified or non-specified components (e.g., system maturity) for which it was not possible to isolate the effect of individual components. Even for evaluations of single interventions, changes in outcome may be due to a connected facet of system resourcing (e.g., helicopter service may be accompanied by better pre-hospital triage or earlier physician intervention). Furthermore, in addition to system-level structures, significant intersystem variation in trauma system outcomes may be explained by injury prevention policies and/or processes of care.

\section{Conclusions}

Injury care is a perfect example of decision-making in conditions of uncertainty and ambiguity, underlying the need for standardized practice guidelines and protocols based on the best available evidence. This review represents a step toward improving our understanding of which components of trauma system structure favor optimal injury outcomes to help policy-makers make informed decisions as to where resources should be focused. Results offer evidence that components focused on getting the right patients to the appropriate facility rapidly are associated with reduced injury mortality and that trauma systems are not fully effective until several years after their implementation. More research is needed on the effectiveness of other recommended components of trauma systems and their impact on non-fatal outcomes using both qualitative and quantitative study designs. Future research should also aim to improve our understanding of the interplay between different components of trauma systems using complex intervention evaluation methodology and care pathway analysis.

Acknowledgements The authors would like to thank Michèle Shemilt for her assistance with the search strategy.

Funding This research is funded by the Fonds de Recherche du Québec-Santé (research career award, LM) and the Canadian Institutes of Health Research (Foundation Grant \#353374 [LM], Canada Research Chair in Critical Care Neurology and Trauma [AFT]).

\section{Compliance with ethical standards}

Conflict of interest The authors declare that they have no conflict of interest.

\section{References}

1. Levit K, Wier L, Stranges E et al (2009) HCUP facts and figures: statistics on hospital-based care in the United States 2007. Agency for Healthcare Research and Quality, Rockville

2. Hofman K, Primack A, Keusch G et al (2005) Addressing the growing burden of trauma and injury in low- and middle-income countries. Am J Public Health 95:13-17

3. Mackenzie EJ, Rivara FP, Jurkovich GJ et al (2007) The national study on costs and outcomes of trauma. J Trauma 63:S54-S67 discussion S81-56

4. Moore L, Turgeon AF, Lauzier F et al (2015) Evolution of patient outcomes over 14 years in a mature, inclusive Canadian trauma system. World J Surg 39:1397-1405. doi:10.1007/s00268-0152977-9

5. Australian Institute of Health and Welfare (2012) The burden of disease and injury in Australia. http://www.aihw.gov.au/burdenof-disease/. Accessed 18 Apr 2016

6. World Health Organisation (2013) WHO global alliance for care of the injured. http://www.who.int/emergencycare/gaci/gaci_ flyer_web.pdf?ua=1\%5d. Accessed 11 May 2016

7. Mock C, Lormand J, Goosen J et al (2004) Guidelines for essential trauma care. http://apps.who.int/iris/bitstream/10665/ 42565/1/9241546409_eng.pdf. Accessed 10 Oct 2016

8. American College of Surgeons Committee on Trauma (2008) Regional trauma systems: optimal elements, integration, and assessment. Systems consultation guide. American College of Surgeons, Chicago

9. Nathens AB, Ball JW, Burton RA et al (2008) Regional trauma systems: optimal elements, integration, and assessment systems consultation guide. In: Surgeons Cotaco (ed). Chicago: American College of Surgeons

10. Celso B, Tepas J, Langland-Orban B et al (2006) A systematic review and meta-analysis comparing outcome of severely injured patients treated in trauma centers following the establishment of trauma systems. J Trauma 60:371-378 discussion 378

11. Gabbe BJ, Biostat GD, Simpson PM et al (2012) Improved functional outcomes for major trauma patients in a regionalized, inclusive trauma system. Ann Surg 255:1009-1015

12. Higgins JPT, Green S (eds) (2011) Cochrane handbook for systematic reviews of interventions, version 5.1.0 [updated March 2011]. The Cochrane Collaboration. Available from www. cochranehandbook.org

13. Shamseer L, Moher D, Clarke M et al (2015) Preferred reporting items for systematic review and meta-analysis protocols (PRISMA-P) 2015: elaboration and explanation. BMJ 349:g7647

14. Moore L, Champion H, O'Reilly G et al (2017) Impact of trauma system structure on injury outcomes: a systematic review protocol. Syst Rev 6:12

15. Lefebvre C, Manheimer E, Glanville J et al (2011) Searching for studies. In: Higgins J, Green S (eds) Cochrane handbook for systematic reviews of interventions. Cochrane Collaboration, London

16. Sterne J, Higgins J, Reeves B (2016) ROBINS-I: a tool for assessing risk of bias in non-randomized studies of interventions. http://www.riskofbias.info. Accessed 25 Mar 2016

17. DerSimonian R, Laird N (1986) Meta-analysis in clinical trials. Control Clin Trials 7:177-188 
18. Berkman ND, Lohr KN, Ansari M, et al (2008) Grading the strength of a body of evidence when assessing health care interventions for the effective health care program of the agency for healthcare research and quality: an update methods guide for effectiveness and comparative effectiveness reviews. Rockville: Agency for Healthcare Research and Quality

19. Carr BG, Wolff CS, Schwab CW et al (2013) The association between geographic access to trauma care and injury death in the us. Acad Emerg Med 20:S313

20. Husum H, Gilbert M, Wisborg T et al (2003) Rural prehospital trauma systems improve trauma outcome in low-income countries: a prospective study from North Iraq and Cambodia. J Trauma Inj Infect Crit Care 54:1188-1196

21. Arreola-Risa C, Mock C, Herrera-Escamilla AJ et al (2004) Costeffectiveness and benefit of alternatives to improve training for prehospital trauma care in Mexico. Prehosp Disaster Med 19:318-325

22. La Banque Mondiale (2014) Médecins (pour 1000 personnes). http://donnees.banquemondiale.org/indicateur/SH.MED.PHYS. ZS. Accessed 10 Sep 2015

23. Cheng $\mathrm{CH}$, Graham CA, Gabbe BI et al (2008) Trauma care systems - a comparison of trauma care in Victoria, Australia, and Hong Kong, China. Ann Surg 247:335-342

24. Nijboer JMM, Wullschleger ME, Nielsen SE et al (2010) A comparison of severely injured trauma patients admitted to level 1 trauma centres in Queensland and Germany. ANZ J Surg $80: 145-150$

25. Haider AH, David JS, Zafar SN et al (2013) Comparative effectiveness of inhospital trauma resuscitation at a French trauma center and matched patients treated in the United States. Ann Surg 258:178-183

26. Timm A, Maegele M, Lefering R et al (2014) Pre-hospital rescue times and actions in severe trauma. A comparison between two trauma systems: Germany and the Netherlands. Injury 45:S43S52

27. Stiell IG, Nesbitt LP, Pickett W et al (2008) The OPALS major trauma study: impact of advanced life-support on survival and morbidity. CMAJ Can Med Assoc $\mathrm{J}=$ journal de l'Association medicale canadienne 178:1141-1152

28. Garner AA, Lee A, Weatherall A (2012) Physician staffed helicopter emergency medical service dispatch via centralised control or directly by crew-case identification rates and effect on the Sydney paediatric trauma system. Scand J Trauma Resusc Emerg Med 20:82

29. Hesselfeldt R, Steinmetz J, Jans H et al (2013) Impact of a physician-staffed helicopter on a regional trauma system: a prospective, controlled, observational study. Acta Anaesthesiol Scand 57:660-668

30. Carr BG, Geiger J, McWilliams $N$ et al (2014) Impact of adding Level II and III trauma centers on volume and disease severity at a nearby Level i trauma center. J Trauma Acute Care Surg 77:764-768

31. Lecky F, Russell W, Fuller G et al (2016) The head injury transportation straight to neurosurgery (HITS-NS) randomised trial: a feasibility study. Health Technol Assess 20:1-198

32. Ali J, Adam RU, Gana TJ, Williams JI et al (1997) Trauma patient outcome after the prehospital trauma life support program. J Trauma 42:1018-1021

33. Rios-Diaz AJ, Metcalfe D, Olufajo OA et al (2016) Geographic distribution of trauma burden, mortality, and services in the United States: Does availability correspond to patient need? J Am Coll Surg 223(764-773):e762

34. Latifi R, Hadeed GJ, Rhee P et al (2009) Initial experiences and outcomes of telepresence in the management of trauma and emergency surgical patients. Am J Surg 198:905-910
35. Utter GH, Maier RV, Rivara FP et al (2006) Inclusive trauma systems: Do they improve triage or outcomes of the severely injured? J Trauma Inj Infect Crit Care 60:529-537

36. Murad MK, Larsen S, Husum H (2012) Prehospital trauma care reduces mortality. Ten-year results from a time-cohort and trauma audit study in Iraq. Scand J Trauma Resusc Emerg Med 20:13

37. He JC, Kreiner LA, Sajankila N et al (2016) Performance of a regional trauma network: a state-wide analysis. J Trauma Acute Care Surg 81:190-195

38. Afifi R (2012) Impact of trauma system preparedness on the outcomes of severe injuries among child populations. Indian $\mathrm{J}$ Surg 74:456-461

39. Deasy C, Gabbe B, Palmer C et al (2012) Paediatric and adolescent trauma care within an integrated trauma system. Injury 43:2006-2011

40. Brown JB, Rosengart MR, Billiar TR et al (2016) Geographic distribution of trauma centers and injury-related mortality in the United States. J Trauma Acute Care Surg 80:42-50

41. Chang DC, Eastman B, Talamini MA et al (2011) Density of surgeons is significantly associated with reduced risk of deaths from motor vehicle crashes in US Counties. J Am Coll Surg 212:862-866

42. Desai A, Bekelis K, Zhao WY et al (2012) Increased population density of neurosurgeons associated with decreased risk of death from motor vehicle accidents in the United States Clinical article. J Neurosurg 117:599-603

43. Moore L, Evans D, Hameed SM et al (2016) Mortality in Canadian trauma systems: a multicenter cohort study. Ann Surg 265:212-217

44. Nathens AB, Jurkovich GJ, Maier RV et al (2001) Relationship between trauma center volume and outcomes. JAMA 285:1164-1171

45. Mohammad A, Branicki F, Abu-Zidan FM (2014) Educational and clinical impact of advanced trauma life support (ATLS) courses: a systematic review. World J Surg 38:322-329. doi:10. 1007/s00268-013-2294-0

46. Brown JB, Gestring ML, Stassen NA et al (2016) Geographic variation in outcome benefits of helicopter transport for trauma in the United States: a retrospective cohort study. Ann Surg 263:406-412

47. Butler DP, Anwar I, Willett K (2010) Is it the H or the EMS in HEMS that has an impact on trauma patient mortality? a systematic review of the evidence. Emerg Med J EMJ 27:692-701

48. Delgado MK, Staudenmayer KL, Wang NE et al (2013) Costeffectiveness of helicopter versus ground emergency medical services for trauma scene transport in the United States. Ann Emerg Med 62(351-364):e319

49. Taylor CB, Stevenson M, Jan S et al (2010) A systematic review of the costs and benefits of helicopter emergency medical services. Injury 41:10-20

50. Galvagno SM, Thomas S, Stephens C et al (2013) Helicopter emergency medical services for adults with major trauma. Cochrane Database Syst Rev 28(3):CD009228. doi:10.1002/ 14651858.CD009228.pub2

51. Center for Disease Control and Prevention (2012) Guidelines for the field triage of injured patients. www.facs.org/ /media/files/ quality\%20programs/trauma/vrc\%20resources/6_guidelines\% 20field\%20triage\%202011.ashx. Accessed 13 Apr 2017

52. Lidal IB, Holte HH, Vist GE (2013) Triage systems for prehospital emergency medical services-a systematic review. Scand J Trauma Resusc Emerg Med 21:28

53. Holmer H, Lantz A, Kunjumen T et al (2015) Global distribution of surgeons, anaesthesiologists, and obstetricians. Lancet Glob Health 3(Suppl 2):S9-11 
54. Meara JG, Leather AJ, Hagander L et al (2015) Global Surgery 2030: evidence and solutions for achieving health, welfare, and economic development. Lancet 386:569-624

55. Hoyler M, Finlayson SR, McClain CD et al (2014) Shortage of doctors, shortage of data: a review of the global surgery, obstetrics, and anesthesia workforce literature. World J Surg 38:269-280. doi:10.1007/s00268-013-2324-y

56. Daniels KM, Riesel JN, Meara JG (2015) The scale-up of the surgical workforce. Lancet 385(Suppl 2):S41

57. WHO emergency and essential surgical care: WHO EESC Global database. http://www.who.int/surgery/eesc_database/en/. Accessed 27 Mar 2017

58. Melton SM, McGwin G Jr, Abernathy JH 3rd et al (2003) Motor vehicle crash-related mortality is associated with prehospital and hospital-based resource availability. J Trauma 54:273-279 discussion 279

59. Vali Y, Rashidian A, Jalili M et al (2017) Effectiveness of regionalization of trauma care services: a systematic review. Public Health 146:92-107

60. Nathens AB, Jurkovich GJ, Cummings P et al (2000) The effect of organized systems of trauma care on motor vehicle crash mortality. JAMA 283:1990-1994

61. Liberman M, Mulder D, Lavoie A et al (2003) Multicenter Canadian study of prehospital trauma care. Ann Surg 237:153-160

62. Schiller J, McCormack JE, Tarsia V et al (2009) The effect of adding a second helicopter on trauma-related mortality in a county-based trauma system. Prehosp Emerg Care 13:437-443

63. Arthur M, Newgard CD, Mullins RJ et al (2009) A populationbased survival assessment of categorizing level III and IV rural hospitals as trauma centers. J Rural Health 25:182-188

64. Hamlat CA, Arbabi S, Koepsell TD et al (2012) National variation in outcomes and costs for splenic injury and the impact of trauma systems: a population-based cohort study. Ann Surg 255:165-170

65. Vanni AJ, Hotaling J, Hamlat C et al (2012) Do inclusive trauma systems improve outcomes after renal trauma? J Trauma Acute Care Surg 72:385-389
66. McKee JL, Roberts DJ, Van Wijngaarden-Stephens MH et al (2015) The right treatment at the right time in the right place: a population-based, before-and-after study of outcomes associated with implementation of an all-inclusive trauma system in a large Canadian province. Ann Surg 261:558-564

67. Simon R, Stone M, Cucuzzo J (2009) The impact of a new trauma center on an existing nearby trauma center. J Trauma Inj Infect Crit Care 67:645-650

68. Crandall M, Sharp D, Wei X et al (2016) Effects of closure of an urban level I trauma centre on adjacent hospitals and local injury mortality: a retrospective, observational study. BMJ Open 6: 5 Article Number: e011700

69. Boyd JM, Moore L, Atenafu EG et al (2017) A retrospective cohort study of the relationship between quality indicator measurement and patient outcomes in adult trauma centers in the United States. Injury 48:13-19

70. Cameron PA, Gabbe BJ, Cooper DJ et al (2008) A statewide system of trauma care in Victoria: effect on patient survival. Med J Aust 189:546-550

71. Moore L, Stelfox HT, Evans D et al (2017) Trends in injury outcomes across Canadian trauma systems. JAMA Surg 152:168-174

72. Moore L, Stelfox H, Evans D et al (2016) Hospital and intensive care unit length of stay for injury admissions: a Pan-Canadian cohort study. Ann Surg. doi:10.1097/SLA.0000000000002036

73. Bakke HK, Wisborg T (2011) Rural high north: a high rate of fatal injury and prehospital death. World J Surg 35:1615-1620. doi:10.1007/s00268-011-1102-y

74. Lipsky AM, Karsteadt LL, Gausche-Hill M et al (2014) A comparison of rural versus urban trauma care. J Emerg Trauma Shock 7:41-46

75. Sampalis JS, Denis R, Lavoie A et al (1999) Trauma care regionalization: a process-outcome evaluation. J Trauma Inj Infect Crit Care 46:565-579 